(C) 2017 IEEE. Personal use of this material is permitted. Permission from IEEE must be obtained for all other uses, in any current or future media, including reprinting/republishing this material for advertising or promotional purposes, creating new collective works, for resale or redistribution to servers or lists, or reuse of any copyrighted component of this work in other works. 


\title{
Eigenspace Time Reversal Robust Capon Beamforming for Target Localization in Continuous Random Media
}

\author{
Md. Delwar Hossain, Member, IEEE and Ananda Sanagavarapu Mohan, Senior Member, IEEE
}

\begin{abstract}
We propose novel Eigenspace Time Reversal Robust Capon Beamformer (E-TR-RCB) for improved target localization in continuous random media. We also derive the Beamspace-TR-RCB (B-TR-RCB) algorithm and compare their localization performances by varying the medium characteristic and excitation bandwidth. FDTD is used to numerically obtain the multistatic scattered field data from the continuous random dielectric medium. The results indicate that the E-TR-RCB has superior performance over the B-TR-RCB and out performs the conventional (elementspace) TR-RCB and DORT imaging techniques.
\end{abstract}

Index Terms-Beamspace, continuous random dielectric medium, DORT, eigenspace, elementspace, localization, microwave imaging, robust Capon beamformer, time reversal.

\section{INTRODUCTION}

$\mathrm{T}$ IME reversal (TR) imaging has already found applications for the localization of targets embedded in random medium [1-8]. For localization in random media, mainly the decomposition of the time reversal operator (DORT) and time reversal MUSIC (TR-MUSIC) techniques or their variations are employed. TR imaging process is carried out in three steps. The first step is the transmission of probing signal towards to the unknown target embedded inside an unknown medium and then record all reflected and backscattered signals from the target and the medium at the receivers. The second step is to time reverse the recorded signals. The third and the crucial step is refocussing of the time reversed received signals into a computational medium which effectively represents the unknown, original medium and it is expected that the refocussed signals converge at the true location of the target. An array synthesis technique for concentrating electromagnetic wave energy into an unknown medium was proposed in $[9,10]$, however, in this paper, we are only interested in using TR as an imaging tool for target localization rather than array synthesis. The interpretation of the TR operator (TRO) as a covariance matrix [11] allows various beamforming techniques such as robust Capon beamformer (RCB) to be implemented for $\mathrm{TR}$ using

This paper was submitted on $17 \mathrm{Jul}$, 2016; revised on 23 Nov, 2016 and accepted on 11 Jan, 2017.

M.D. Hossain and A.S. Mohan are with the Centre for Health Technologies, University of Technology Sydney (UTS), NSW 2007, Australia (e-mail: Md.Hossain@uts.edu.au, Ananda.Sanagavarapu@uts.edu.au). multistatic measurement data. The DORT and TR-MUSIC are subspace based techniques that require eigen decomposition of the TRO into two orthogonal signal and noise subspaces. On the other hand, the TR-RCB method estimates the array weight vectors [12] which are robust against mismatch of medium Green's function for TR refocusing step. The conventional, elementspace TR-RCB has already been explored for biomedical imaging applications for the localization of embedded targets in biological tissues [13, 14]. In this study, we consider single target localization in a continuous random medium. In conventional TR-RCB, the imaging is mainly performed in elementspace whereby the output data from individual sensor elements is directly used in the imaging process. The elementspace processing usually leads to high intensity clutter spots and thus, resulting in location ambiguities. Hence, we require techniques that can improve over the elementspace TR-RCB imaging performance.

In this paper, we propose Eigenspace-TR-RCB (E-TRRCB) and Beamspace-TR-RCB (B-TR-RCB) algorithms for improved localization performance in continuous random dielectric media. The E-TR-RCB can be obtained by projecting the array weight vector onto the eigenspace of the TRO so that the eigenspace is orthogonal to the noise subspace of the TRO thereby significantly reducing the clutter signature and improve resolution. Eigenspace processing also improves convergence and reduces error sensitivity of the $\mathrm{RCB}[15,16]$. On the other hand, the B-TR-RCB is derived by projecting the elementspace multistatic data onto the beamspace to potentially reduce computational complexity, improve resolution threshold and increase robustness against sensor perturbations $[8,17]$. To the best of our knowledge, neither the beamspace nor the eigenspace versions of TR-RCB have so far been reported in the literature.

The paper is organized as follows. In section II, we discuss the formulation of the proposed B-TR-RCB and E-TR-RCB. The numerical formulation for electromagnetic scattering from 2 -D continuous random media is provided in section III. The numerical results and analysis are presented in section IV followed by conclusion in section $\mathrm{V}$.

\section{TR-RCB IMAGING}

Consider an antenna array consisting of $N$ elements that radiates into a 2-D bounded region containing $P(P<N)$ 
infinitesimal targets. The $N \times N$ multistatic matrix of the medium formed using distorted Born approximation can be expressed as

$$
\mathbf{K}=\mathbf{G} \mathbf{Y} \mathbf{G}^{T}
$$

where, $(.)^{T}$ denotes transpose, $\mathbf{G}$ is an $N \times P$ matrix of the background medium's Green's function vector, $\mathbf{Y}$ and is a $P \times P$ diagonal matrix representing the scattering strengths of the targets. Thus, the TRO, T can be obtained as [13]

$$
\mathbf{T}=\mathbf{K K}^{H}
$$

where, $(.)^{H}$ denotes Hermitian transpose.

\section{A. Elementspace TR-RCB}

In elementspace TR-RCB, the raw data recorded at each element of the array is processed separately. A robust estimation of the array weight vector is obtained by minimizing the noise variance, which can be simplified as

$$
\min _{\mathbf{g}} \mathbf{g}^{H} \mathbf{T}^{-1} \mathbf{g} \quad \text { subject to }\|\mathbf{g}-\tilde{\mathbf{g}}\|^{2} \leq \varphi
$$

where, $\tilde{\mathbf{g}}$ is the background medium's Green's function vector and $\varphi \geq 0$. We can solve (3) using Lagrange multiplier methodology to estimate $\mathbf{g}$ and obtain its scaled version based on constant norm assumption as $\hat{\mathbf{g}}=\mathbf{g}\|\tilde{\mathbf{g}}\| /\|\mathbf{g}\|$ [13]. The TR$\mathrm{RCB}$ array weight vector in elementspace is given by

$$
\mathbf{w}=\frac{\mathbf{T}^{-1} \hat{\mathbf{g}}}{\hat{\mathbf{g}}^{H} \mathbf{T}^{-1} \hat{\mathbf{g}}}
$$

Finally, the TR-RCB imaging function in elementspace is obtained as

$$
\mathcal{P}_{T R-R C B}=\mathbf{w}^{H} \mathbf{T} \mathbf{w}
$$

\section{B. Beamspace-TR-RCB}

In Beamspace TR-RCB, the output at each antenna array element is first applied to a beamformer whose output will be processed further for imaging. The space spanned by the beamformer output is called beamspace. Thus, an additional beamforming gain is provided which improves the imaging performance as compared to elementspace. We employ full dimensional beamspace in the receiving mode using conventional DFT beamformer expressed as

$$
[\mathbf{B}]_{m n}=\frac{e^{j 2 \pi \alpha_{m} n / N}}{N}
$$

where, $\alpha_{m}=m-(N-1) / 2, \mathrm{~m}=0,1, \ldots, \quad(N-1)$. This is equivalent to spatial sampling of the incoming waveforms and the beamspace multistatic matrix can be expressed as

$$
\mathbf{K}_{B}=\mathbf{B}^{H} \mathbf{K}
$$

Consequently, the beamspace TRO is formed as

$$
\mathbf{T}_{B}=\mathbf{K}_{B} \boldsymbol{K}_{B}^{H}=\mathbf{B}^{H} \mathbf{K K}^{H} \mathbf{B}=\mathbf{B}^{H} \mathbf{T B}
$$

The beamspace projection of the Green's function vector can be obtained as

$$
\tilde{\mathbf{g}}_{B}=\left[\mathbf{B}^{H} \mathbf{B}\right]^{-\mathbf{1}} \mathbf{B}^{H} \tilde{\mathbf{g}}=\mathbf{B}^{H} \tilde{\mathbf{g}}
$$

In order to avoid any trivial solution $\mathbf{g}_{B}=0$, we assume that $\left\|\tilde{\mathbf{g}}_{B}\right\|>\varphi$. The Lagrange multiplier, $\gamma \geq 0$ for B-TR-RCB imaging can be obtained from

$$
\left\|\left(\mathbf{I}+\gamma \mathbf{T}_{B}\right)^{-\mathbf{1}} \tilde{\mathbf{g}}_{B}\right\|^{2}-\varphi=0
$$

Now, an estimation of $\mathbf{g}_{B}$ can be expressed as

$$
\mathbf{g}_{B}=\tilde{\mathbf{g}}_{B}-\left(\mathbf{I}+\gamma \mathbf{T}_{B}\right)^{-\mathbf{1}} \tilde{\mathbf{g}}_{B}
$$

To further avoid trivial solution in beamspace domain, scaling of $\mathbf{g}_{B}$ is carried out using

$$
\hat{\mathbf{g}}_{B}=\frac{\mathbf{g}_{B}\left\|\tilde{\mathbf{g}}_{B}\right\|}{\left\|\mathbf{g}_{B}\right\|}
$$

The imaging function of B-TR-RCB is given by

$$
\mathcal{P}_{B-T R-R C B}=\mathbf{w}_{B}^{H} \mathbf{T}_{B} \mathbf{w}_{B}
$$

where $\mathbf{w}_{B}$ can be obtained by substituting (8) and (12) into (4).

\section{Eigenspace- TR-RCB}

Eigenspace TR-RCB processing is carried out by projecting the elementspace data onto a reduced dimensional subspace called the eigenspace. The vector space of TRO, $\boldsymbol{C}^{N}$ can be expressed as the direct sum of orthogonal signal subspace, $\boldsymbol{S}$ and noise subspace, $\mathcal{N}$ i.e. $\boldsymbol{C}^{N}=\boldsymbol{S} \oplus \mathcal{N}$ where $\boldsymbol{S} \perp \mathcal{N}$. The two subspaces can be obtained through eigen decomposition of the TRO.

$$
\mathbf{T}=\sum_{n=1}^{N} \lambda_{n} \mathbf{v}_{n} \mathbf{v}_{n}^{H}=\mathbf{V}_{\mathcal{S}} \boldsymbol{\Lambda}_{\mathcal{S}} \mathbf{V}_{\mathcal{S}}^{H}+\mathbf{V}_{\mathcal{N}} \boldsymbol{\Lambda}_{\mathcal{N}} \mathbf{V}_{\mathcal{N}}^{H}
$$

Here, $\boldsymbol{\Lambda}_{\mathcal{S}}$ is a diagonal matrix of the significant eigen values $\lambda_{1} \geq \lambda_{2} \geq \cdots \geq \lambda_{P}$ and $\boldsymbol{\Lambda}_{\mathcal{N}}$ is a diagonal matrix of the insignificant eigen values $\lambda_{P+1} \approx \lambda_{P+2} \approx \cdots \approx \lambda_{N} \approx \eta^{2}, \eta^{2}$ being the noise variance of additive white Gaussian noise (AWGN) in the medium. The columns of $\mathbf{V}_{\mathcal{S}}$ span the signal subspace and the columns of $\mathbf{V}_{\mathcal{N}}$ span the noise subspace.

The corresponding array weight vector can be expressed as

$$
\mathbf{w}=\mu\left(\mathbf{T}^{-1} \hat{\mathbf{g}}\right)=\mu\left(\mathbf{V}_{\mathcal{S}} \boldsymbol{\Lambda}_{\mathcal{S}}^{-1} \mathbf{V}_{\mathcal{S}}^{H}+\mathbf{V}_{\mathcal{N}} \boldsymbol{\Lambda}_{\mathcal{N}}^{-1} \mathbf{V}_{\mathcal{N}}^{H}\right) \hat{\mathbf{g}}
$$

where, $\mu=1 /\left(\hat{\mathbf{g}}^{H} \mathbf{T}^{-\mathbf{1}} \hat{\mathbf{g}}\right)$. The Green's function vector forms the orthonormal basis for the signal subspace eigen vectors which are in turn, orthogonal to the noise subspace eigen vectors. This makes the contribution from the product of noise subspace and the Green's function vector is insignificant in (14). Recognizing this and rearranging (14), we obtain the eigenspace array weight vector as its projection onto the eigenspace of the TRO as

$$
\mathbf{w}_{E}=\mathbf{V}_{\mathcal{S}} \mathbf{V}_{\mathcal{S}}^{H} \mathbf{w}
$$

We can then obtain E-TR-RCB imaging function by substituting the weight vector from (15) into (5).

\section{EM SCATTERING FROM CONTINUOUS RANDOM MEDIUM}

Here 2-D FDTD technique is used with PML absorbing boundary to calculate the scattered fields from a 2-D continuous random medium whose dielectric variation has a Gaussian profile. The spatial dielectric variations in the medium can be expressed as $\varepsilon(\mathbf{r})=\varepsilon_{m}(\mathbf{r})+\varepsilon_{\sigma}(\mathbf{r})$, where $\varepsilon_{m}$ is the mean dielectric constant and $\varepsilon \sigma(\mathbf{r})$ is a zero mean Gaussian random variable characterized by its standard deviation and spatial correlation length [18]. The two different realizations of such continuous random medium used in this study are shown in Fig. 1. In both the cases we fix $\varepsilon_{m}=5.5$. However, the spatial variance of the dielectric constant, $\varepsilon_{\sigma}$ is $0.075 \varepsilon_{m}$ for Fig. 1(a) and $0.15 \varepsilon_{m}$ for Fig. 1(b). The correlation lengths are $0.4 \mathrm{~m}$ and $0.8 \mathrm{~m}$ respectively. The medium in Fig. 1(a) is excited using two differentiated Gaussian pulses: (i) a center frequency $0.65 \mathrm{GHz}$ and $3-\mathrm{dB}$ bandwidth of $0.3-1.0 \mathrm{GHz}$ and (ii) center frequency $0.325 \mathrm{GHz}$ with a $3-\mathrm{dB}$ bandwidth $0.15-0.5 \mathrm{GHz}$ mainly to investigate effects of excitation pulse.

We consider the random medium to be lying along the $x y$ plane and employ TM-z polarization. We employ a 15 element uniform linear array (ULA) of line sources with an element 
spacing of $0.05 \mathrm{~m}$ to record the backscattered field data from the medium. The locations of the array elements are indicated by the black markers in Fig. 1. The FDTD grid size is $\Delta=5 \mathrm{~mm}$ and a dielectric target of radius $5 \mathrm{~cm}$ is inserted at $x=0.25 \mathrm{~m}, y=1.25 \mathrm{~m}$ for the case in Fig. 1(a) and $x=-0.4 \mathrm{~m}, y=1.5 \mathrm{~m}$ for the case in Fig. 1(b). The scattered field is obtained by subtracting the incident field from the total field. The incident field refers to the field received in a homogeneous medium where the dielectric constant is the mean permittivity of the random medium [18].

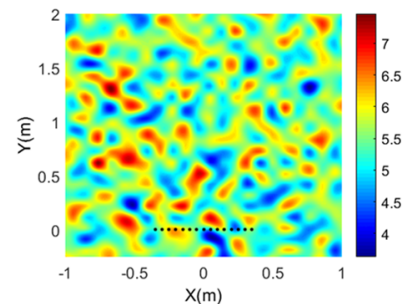

(a) $\varepsilon_{m}=5.5, \varepsilon \sigma=0.075 \varepsilon_{m}, l_{c}=0.4 \mathrm{~m}$

Fig. 1. Continuous random medium.

\section{RESULTS AND ANALYSIS}

For the refocusing of time reversed fields, an equivalent computational, homogeneous background medium with a known Green's function is required. To compute the Green's function, it is assumed that the computational homogeneous medium represents an average equivalent property of the original random medium and further we assume that the mean dielectric constant of the original random medium is known [2].

Let us first consider the medium in Fig. 1(a) with the first excitation having a $3-\mathrm{dB}$ bandwidth $0.3-1.0 \mathrm{GHz}$. The results are shown in Figs. 2(a)-(d) where all the images are normalized with respect to the highest imaging intensity whose dynamic level is adjusted from 0 to $-15 \mathrm{~dB}$ for ease of comparison. The estimated locations by both TR-RCB and BTR-RCB fall close to the edge of the inserted dielectric target as indicated by the highest intensity images. One can also observe presence of bright clutter spots in in Fig. 2(a) due to elementspace TR-RCB but reduced clutter spot intensity is observed due to the use of B-TR-RCB in Fig. 2(b). The image obtained by the E-TR-RCB is shown in Fig. 2(c), in which not only the correct location of the target is estimated but also significant reduction of the clutter intensity. This is due to the effective elimination of the noise-subspace contribution by the eigenspace processing. When eigenspace projection of the array weight vector is used for E-TR-RCB, nulls are produced for corresponding clutters and as a result, the clutter spots are highly suppressed in E-TR-RCB image. For comparison sake, as shown in Fig. 2(d), we also compute image using DORT technique since it is robust against clutter although its resolution is low.

We also observe similar trends when the second excitation pulse with a $3-\mathrm{dB}$ bandwidth $0.15-0.5 \mathrm{GHz}$ is used. However, in this case, we observe a slight deterioration of the resolution which is expected with the use of lower frequency band. Now we consider the medium in Fig. 1(b) with higher variance and correlation length of the dielectric constant. The imaging results are provided in Fig. 3 for the first excitation pulse having a 3-dB bandwidth $0.3-1 \mathrm{GHz}$. In this case also, we observe that the E-TR-RCB outperforms both TR-RCB and B-TR-RCB techniques. However, when the same excitation pulse bandwidth as used in Figs. 2(a)-(d) is employed, we observe wider main lobe as can be seen in Figs. 3(a)-(d). This can arise due to higher variance of the dielectric constant variation of the medium in Fig. 1(b) as compared to the medium considered in Fig. 1(a). However, the clutter spot intensity is weaker in Fig. 3 when compared to Fig. 2. This is the consequence of large correlation length of the medium as used in Fig. 1(b) as compared to that of Fig. 1(a).

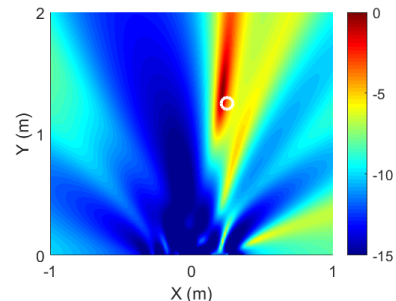

(a) TR-RCB,

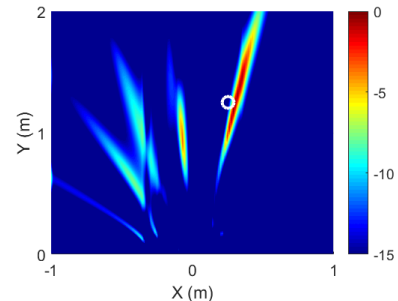

(b) B-TR-RCB

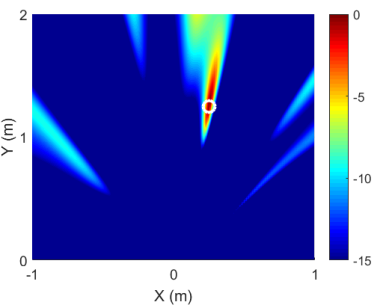

(c) E-TR-RCB

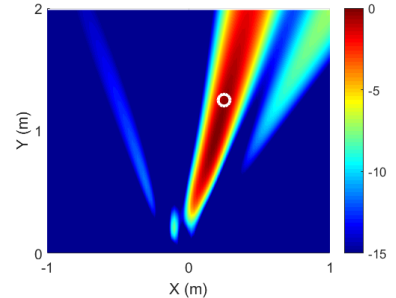

(d) DORT

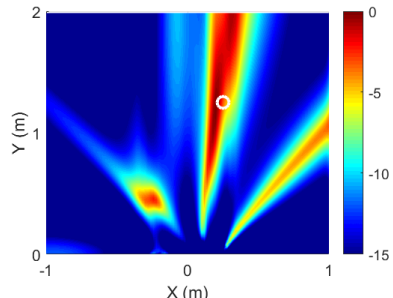

(e) TR-RCB

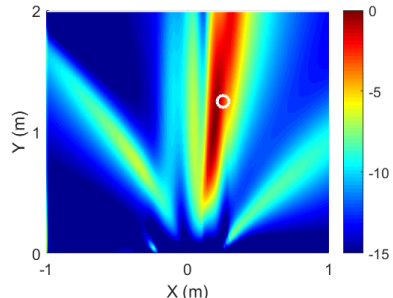

(f) B-TR-RCB

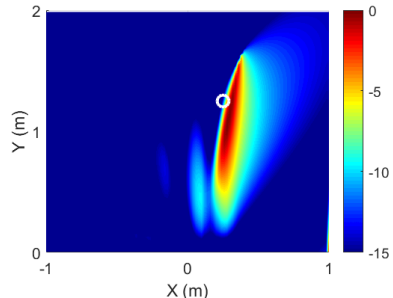

(g) E-TR-RCB

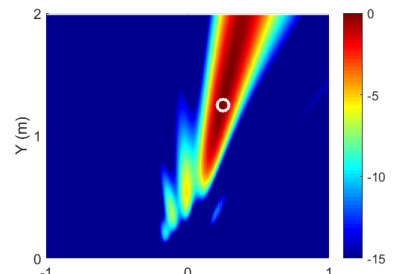

(h) DORT
Fig. 2. Target localization results for the continuous random medium in Fig. 1(a).

The cross range comparison among the imaging techniques as shown in Fig.4, readily confirms the superiority of E-TRRCB. In Fig.4, the center of the target is indicated using the black vertical line. We also compute peak to sidelobe ratio (PSLR) for all the images in Figs. 2-3 in order to provide a clear comparison among DORT, TR-RCB and the proposed B-TR-RCB, E-TR-RCB as tabulated in Table I. In all the cases, we observe that E-TR-RCB yields superior images while B-TR-RCB obtains lower clutter spot luminosity than conventional, elementspace TR-RCB. DORT technique although robust, suffers from poor range resolution which may create ambiguity regarding precise location of the target. The 
E-TR-RCB on the other hand shows a highly focused bright intensity at the target location.

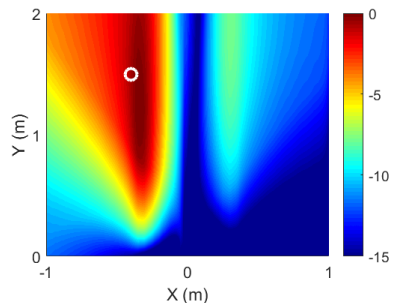

(a) TR-RCB

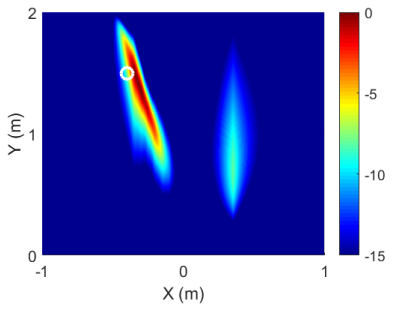

(b) B-TR-RCB

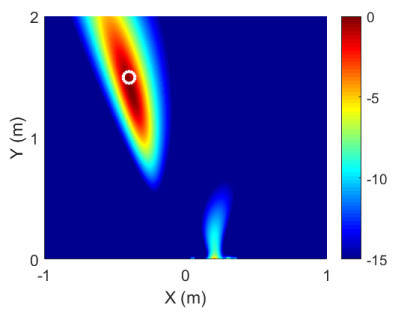

(c) E-TR-RCB

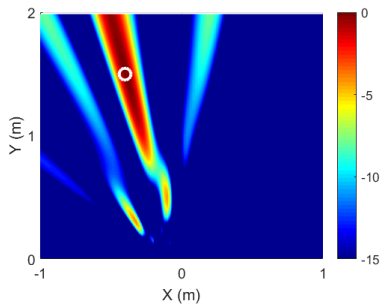

(d) DORT
Fig. 3. Target localization results for continuous random medium in Fig. 1(b).

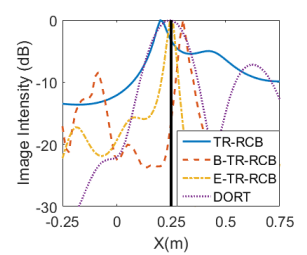

(a) Figs. 2(a)-(d)

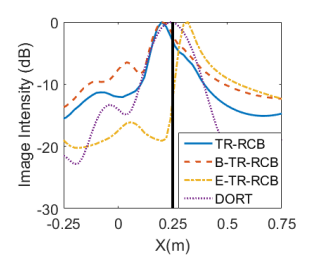

(b) Figs. 2(e)-(h)

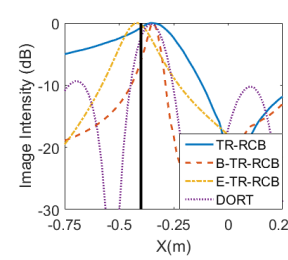

(c) Figs. 3(a)-(d)
Fig. 4. Cross range comparison.

In order to investigate the effect of medium mismatch, we also carried out imaging where the homogeneous computational medium permittivity vary up to $50 \%$ of the mean permittivity of the random medium. It is found that ETR-RCB is quite robust while TR-RCB is the most affected among the imaging techniques considered here. It is also observed that the TR image focal point shifts away from the antenna array with increasing dielectric constant of the computational medium.

\section{CONCLUSIONS}

We have proposed E-TR-RCB and B-TR-RCB for localizing dielectric targets embedded in a 2-D continuous random dielectric medium. Numerical results are obtained with different medium characteristics and excitation pulses clearly demonstrate that the proposed E-TR-RCB and B-TR$\mathrm{RCB}$ significantly improve the imaging performance over the conventional TR-RCB and DORT imaging techniques. Evidently, E-TR-RCB outperforms the other localization techniques considered here in terms of accuracy, resolution and PSLR for targets embedded in continuous random media. Although a single target is considered in this paper, our technique can be extended for multiple targets without any loss of generality provided that there are sufficient degrees of freedom available. In such scenarios, compressive sensing can be quite attractive to exploit the resulting signal sparsity in order to reduce computational burden [19].
TABLE I

PSLR (DB) COMPARISON OF DIFFERENT IMAGING TECHNIQUES

\begin{tabular}{l|c|c|c|c}
\hline Medium & TR-RCB & B-TR-RCB & E-TR-RCB & DORT \\
\hline Fig. 1(a)* & 5.12 & 5.47 & 6.80 & 6.39 \\
Fig. 1(a) & 2.25 & 6.50 & 9.53 & 5.33 \\
Fig. 1(b)* & 7.91 & 8.10 & 9.15 & 4.05 \\
\hline \hline
\end{tabular}

Excitation bandwidth *0.3-1GHz, ${ }^{*} 0.15-0.5 \mathrm{GHz}$.

\section{REFERENCES}

[1] M. E. Yavuz and F. L. Teixeira, "Full time-domain DORT for ultrawideband electromagnetic fields in dispersive, random inhomogeneous media," IEEE Trans. Antennas Propag., vol. 54, pp. 2305-2315, 2006.

[2] M. E. Yavuz and F. L. Teixeira, "Space frequency ultrawideband timereversal imaging," IEEE Trans. Geosci. Remote Sens., vol. 46, pp. 1115$1124,2008$.

[3] Y. Ziade, H. Roussel, M. Lesturgie, and W. Tabbara, "A coherent model of forest propagation-application to detection and localization of targets using the DORT method," IEEE Trans. Antennas Propag., vol. 56, pp. 1048-1057, 2008.

[4] A. E. Fouda, V. Lopez-Castellanos, and F. L. Teixeira, "Experimental demonstration of statistical stability in ultrawideband time-reversal imaging," IEEE Geosci. Remote Sens. Lett., vol. 11, pp. 29-33, 2014.

[5] A. E. Fouda and F. L. Teixeira, "Statistical stability of ultrawideband time-reversal imaging in random media," IEEE Trans. Geosci. Remote Sens., vol. 52, pp. 870-879, 2014.

[6] X. Zhong, C. Liao, and W. Lin, "Space-frequency decomposition and time-reversal imaging," IEEE Trans. Antennas Propag., vol. 63, pp. 5619-5628, 2015

[7] D. A. M. Iero, T. Isernia, and L. Crocco, "Focusing time-harmonic scalar fields in complex scenarios: a comparison," IEEE Antennas Wilreless Propag. Lett., vol. 12, pp. 1029-1032, 2013.

[8] M. D. Hossain, A. S. Mohan, and M. J. Abedin, "Beamspace timereversal microwave imaging for breast cancer detection," IEEE Antennas Wilreless Propag. Lett., vol. 12, pp. 241-244, 2013.

[9] L. Crocco, L. D. Donato, and D. A. M. Iero, "A new strategy to constrained focusing in unknown scenarios," IEEE Antennas Wireless Propag. Lett., vol. 11, pp. 1450 - 1453, 2012.

[10] L. Crocco, L. D. Donato, D. A. M. Iero, and T. Isernia, "An adaptive method to focusing in an unknown scenario," Prog. Electromagn. Res., vol. 130, pp. 563-579, 2012.

[11] C. Prada and J.-L. Thomas, "Experimental subwavelength localization of scatterers by decomposition of the time reversal operator interpreted as a covariance matrix," J. Acoust. Soc. Am., vol. 114, pp. 235-243, 2003.

[12] P. Stoica, Z. Wang, and J. Li, "Robust Capon beamforming," IEEE Signal Process. Lett., vol. 10, pp. 172-175, 2003.

[13] Z. Wang, J. Li, and R. Wu, "Time-delay- and time-reversal-based robust capon beamformers for ultrasound imaging," IEEE Trans. Med. Imag., vol. 24, pp. 1308-1322, 2005.

[14] M. D. Hossain and A. S. Mohan, "Coherent time-reversal microwave imaging for the detection and localization of breast tissue malignancies," Radio Science, vol. 50, pp. 87-98, 2015.

[15] B. M. Asl and A. Mahloojifar, "Eigenspace-based minimum variance beamforming applied to medical ultrasound imaging," IEEE Trans. Ultrason. Ferroelectr. Freq. Control, vol. 57, pp. 2381-2390, 2010.

[16] S. Mehdizadeh, A. Austeng, T. F. Johansen, and S. Holm, "eigenspace based minimum variance beamforming applied to ultrasound imaging of acoustically hard tissues," IEEE Trans. Med. Imag., vol. 31, pp. 19121921, 2012.

[17] H. L. V. Trees, Optimum Array Processing: Part IV of of Detection, Estimation, and Modulation Theory. New York, USA: John Wiley \& Sons, 2002.

[18] C. D. Moss, F. L. Teixeira, Y. E. Yang, and J. A. Kong, "Finitedifference time-domain simulation of scattering from objects in continuous random media," IEEE Trans. Geosci. Remote Sens., vol. 40, pp. 178-186, 2002.

[19] M. H. S. Sajjadieh and A. Asif, "Compressive sensing time reversal MIMO radar: joint direction and doppler frequency estimation," IEEE Signal Process. Lett., vol. 22, pp. 1283-1287, 2015. 